\title{
Desarrollo de una Aplicación Móvil para la eficiente gestión de sustancias Químicas con base en la Norma Oficial Mexicana NOM-018-STPS-2015
}

\section{Development of a Mobile Application for an efficient the management of Chemical substances based on the Official Mexican Norm NOM-018-STPS-2015}

\author{
ARCIGA-PEDRAZA, Raquel†*, FUENTES-URIBE, Luis Ángel, JUAREZ-SANTIAGO, Brenda y \\ MARTINEZ-MIRANDA, Erick
}

Universidad Tecnológica de San Juan del Río

ID $1^{\mathrm{er}}$ Autor: Raquel, Arciga-Pedraza / ORC ID: 0000-0003- 1163-4693, Researcher ID Thomson: G-3359-2019

ID $1^{\text {er }}$ Coautor: Luis Ángel, Fuentes-Uribe / ORC ID: 0000-0002-8117-9369, Researcher ID Thomson: G-2116-2019

ID $2^{\text {do }}$ Coautor: Brenda, Juarez-Santiago / ORC ID: 0000-0001-9071-9243, Researcher ID Thomson: F-7396-2017

ID $3^{\text {er }}$ Coautor: Erick, Martinez-Miranda / ORC ID: 0000-0002-3270-8076, Researcher ID Thomson: G-2617-2019

DOI: $10.35429 / J C A .2019 .12 .3 .29 .41$

Recibido Abril 30, 2019; Aceptado Junio 30, 2019

\begin{abstract}
Resumen
Actualmente el uso de las aplicaciones móviles ha contribuido en las diferentes áreas de la ingeniería, el proyecto que se presenta es el desarrollo de una aplicación móvil para el área de Ingeniería Química, que permitió la gestión eficaz de los reactivos bajo la Norma Oficial Mexicana NOM-018-STPS-2015. La metodología utilizada para el de desarrollo de la aplicación es Scrum; la cual es una Framework ágil que permite la colaboración, auto-organización, entrega temprana, transparencia, entre otros beneficios; el grupo de trabajo se conformó por estudiantes y docentes de la división de Tecnologías de la Información y la Comunicación y la división de Química Industrial y Farmacéutica. La iniciativa de implementar una aplicación móvil para dispositivos de sistema operativo Android, con una API mayor o igual a 4.4, permitió tener una solución altamente eficaz, optimizando la gestión en el manejo de sustancias químicas a utilizar por alumnos y docentes, agilizando el proceso de entrega de insumos de laboratorio y garantizando el cumplimiento del proceso de aprendizaje en los laboratorios de la Universidad Tecnológica de San Juan del Río, se implementó con alumnos de ingeniería Química Industrial y Farmacéutica, donde $86 \%$ de los usuarios afirmaron que la aplicación resolvió el problema.
\end{abstract}

Scrum, NOM-018-STPS-2015, Software

\begin{abstract}
Nowadays the use of mobile applications has contributed in different areas of engineering, the following project presents the development of a mobile application for the chemistry area, which allowed the effective management of substances following the Official Mexican Norm NOM-018-STPS-2015. The utilized method for the development of the application is scrum; which is an agile framework that allows collaboration, self-organization, early delivery, transparency beyond others; the workgroup was formed by students and teachers of the divisions of technologies of the information and communication and pharmaceutical and industrial chemistry. The initiative of implementing a mobile application for devices with an android operating system, with an API greater or equal to 4.4 allowed a highly effective, optimizing the management of the control of chemical substances used by students and teachers, Streamlining the process of returning the laboratory supplies and guaranteeing the fulfillment of the learning process in the laboratories of the technological university of San Juan Del Rio, it was implemented with students of Industrial and pharmaceutical chemistry engineering, where $86 \%$ of the users affirmed to be satisfied with the mobile application.
\end{abstract}

Scrum, NOM-018-STPS-2 015, Software

Citación: ARCIGA-PEDRAZA, Raquel, FUENTES-URIBE, Luis Ángel, JUAREZ-SANTIAGO, Brenda y MARTINEZMIRANDA, Erick. Desarrollo de una Aplicación Móvil para la eficiente gestión de sustancias Químicas con base en la Norma Oficial Mexicana NOM-018-STPS-2015. Revista de Cómputo Aplicado. 2019, 3-12: 29-41

\footnotetext{
* Correspondencia al Autor (Correo electrónico: raicigap@utsjr.edu.mx)

$\dagger$ Investigador contribuyendo como primer Autor.
} 


\section{Introducción}

El presente proyecto surgió a causa de la actualización de la norma oficial mexicana NOM-018-STPS-2000 a la nueva norma oficial mexicana NOM-018-STPS-2015, sistema armonizado para la identificación y comunicación de peligros y riesgos por sustancias químicas peligrosas en los centros de trabajo.

Publicada el 9 de octubre de 2018 en el Diario Oficial de la Federación (DOF), la cual propone una reingeniería total sobre el método tradicional que se utilizaba para el manejo de sustancias químicas de los laboratorios, dicha norma era utilizada en los laboratorios de la Universidad Tecnológica de San Juan del Río (UTSJR), por ello es que al ser actualizada, se requiere de un sistema que permita gestionar las sustancias químicas existentes en almacén y utilizadas en el proceso de aprendizaje de dicha Institución basado en la nueva norma.

Actualmente la UTSJR utiliza hojas de cálculo y bitácoras para llevar a cabo la gestión de dichas sustancias por lo que el proceso es lento, la integridad, disponibilidad, portabilidad y otros factores relacionados con el manejo de datos están casi ausentes.

Por lo anterior es que surge la iniciativa de docentes y alumnos de la división de Química Industrial y Farmacéutica, así como de Tecnologías de la Información y la Comunicación (TIC) de trabajar en conjunto para realizar un sistema que sea innovador, eficiente, portable, con alto grado de disponibilidad e integridad de los datos y que sea capaz de resolver la problemática.

El desarrollo de una aplicación que permita la gestión de sustancias químicas a través de una interfaz gráfica y al alcance de un clic en un dispositivo móvil es una gran noticia y de suma importancia para laboratoristas, estudiantes, encargados de almacén y cualquier miembro de la comunidad química-científica, puesto que la tediosa tarea de crear hojas de cálculo, bitácoras o en el peor de los casos anotaciones en libretas para tener un "control" simplemente está por terminar.
El valor agregado de la aplicación o lo que la hace realmente interesante es que surgió de una necesidad relativamente joven por lo que actualmente no existen aplicaciones que realicen lo mismo, es decir es una innovación que surgió a causa de la implementación de la NOM-018STPS-2015.

El problema que se pretende solucionar a corto plazo es la inexistencia de un sistema de información que permita la gestión de sustancias químicas de los laboratorios de la UTSJR, esperando proveer una solución tecnológica e innovadora basada en los lineamientos impuestos por la norma NOM-018-STPS-2015 reduciendo los tiempos que demanda la ejecución de dichos procesos y al mismo tiempo incrementando la portabilidad, disponibilidad e integridad de los datos.

\section{Planteamiento del problema}

A causa de la última actualización de la norma oficial mexicana NOM-018-STPS-2015 la forma en como las sustancias químicas son clasificadas se modificó, por lo que los laboratorios de química industrial y farmacéutica de la UTSJR requieren de un nuevo sistema que permita gestionar las sustancias existentes con base en los nuevos lineamientos que estableció dicha norma y con algunas características especiales como registrar la cantidad existente de cada sustancia de una manera más fácil, rápida y sencilla.

\section{Justificación}

Tras la última actualización de la norma oficial mexicana NOM-018-STPS-2015, sistema armonizado para la identificación y comunicación de peligros y riesgos por sustancias químicas peligrosas en los centros de trabajo, publicada el 9 de octubre del pasado año 2018 DOF, la forma en cómo se gestionan las sustancias químicas peligrosas ha sido modificada, por ello, la UTSJR no contaba con un sistema de información que agilizara los procesos de gestión de sustancias con base en los lineamientos que rige dicha norma, obligando a encargados de laboratorio y docentes a realizar el proceso manualmente en bitácoras, libretas y hojas de cálculo, siendo un proceso que consumía bastante tiempo, además que factores como disponibilidad, e integridad de datos estaban casi ausentes. 
Por lo anterior y detrás de un largo análisis se decidió entre docentes y alumnos de TIC y Química industrial y farmacéutica, desarrollar una aplicación móvil para Smartphones de sistema operativo Android que permita a laboratoristas y docentes realizar el proceso de registro y consulta de información de las sustancias químicas, almacenadas en los laboratorios de la UTSJR de una manera mucho más práctica, eficiente, y con elevados niveles de disponibilidad, portabilidad e integridad de datos.

\section{Objetivo general}

Desarrollar una aplicación móvil para Smartphones de sistema operativo Android que permita la inserción, modificación, eliminación y consulta de sustancias químicas con base en la Norma oficial mexicana NOM-018-STPS-2015 para facilitar el proceso de gestión de las mismas.

\section{Metodología}

La metodología utilizada para el desarrollo de la presente aplicación móvil es una de las más usadas dentro del ámbito del desarrollo de software, llamada Scrum, metodología ágil que permitió optimizar los procesos internos de desarrollo que fueron aplicados y obtener una entrega temprana de alto valor del producto final. Scrum garantiza transparencia en la comunicación y crea un ambiente de responsabilidad colectiva y de progreso continuo. El framework de Scrum está estructurado de tal manera que es compatible con el desarrollo de productos y servicios en todo tipo de industrias y en cualquier tipo de proyecto, independientemente de su complejidad. Beneficios obtenidos al utilizar la metodología Scrum:

1. Adaptabilidad - El control del proceso empírico y el desarrollo iterativo fueron adaptables y abiertos a la incorporación del cambio.

2. Se llevaron a cabo Sprints con una duración de un mes aproximadamente cada uno.
3. Transparencia - Todos los radiadores de información tales como un Scrumboard y el Sprint Burndown Chart se compartieron, lo cual condujo a un ambiente de trabajo abierto. Retroalimentación continua - La retroalimentación continua fue proporcionada a través de los procesos de Realizar Daily Standup y Demostrar $y$ validar el sprint.

Todos los días el equipo de desarrollo llevaba a cabo una reunión diaria de 15 minutos donde se discutían las tareas realizadas, que había por hacer en ese día y además si existían problemas para poder resolverlos.

4. Mejora continua - Los entregables mejoraron progresivamente sprint por sprint a través del proceso de Refinar el Backlog Priorizado del Producto.Los entregables fueron mejorando Sprint por Sprint ya que al término de cada uno se mostraban los entregables al cliente y podían existir posibles cambios, los cuales se agregaban nuevamente al Backlog Priorizado del producto.

5. Entrega continúa de valor - Los procesos iterativos permitieron la entrega continua de valor tan frecuentemente como el cliente lo requiere a través del proceso de Envío de entregables.

La entrega continua de valor se realizaba a final de cada Sprint donde los entregables o pruebas funcionales se mostraban al cliente para verificar que existía un proceso de desarrollo.

6. Entrega anticipada de alto valor - El proceso de Crear el Backlog Priorizado del Producto aseguro que los requisitos de mayor valor del cliente fueran los primeros en cumplirse.

7. Resolución de problemas de forma más rápida - La colaboración y co-ubicación de equipos interfuncionales condujeron a la resolución de problemas con mayor rapidez. 
La manera de desarrollar el proyecto con una alta comunicación y transparencia del equipo ayudaba a la resolución de problemas de una manera más rápida por que el equipo siempre permanecía junto permitiendo resolver los problemas entre todo el equipo.

8. Entregables efectivos - El proceso de Crear el Backlog Priorizado del Producto, y las revisiones periódicas después de la creación de entregables aseguraron entregas eficientes al cliente.

El entregar los resultados al cliente en cada Sprint y recibir retroalimentación ayudo a asegurar entregas de alta calidad.

9. Alta velocidad - La ventaja de trabajar con la metodología Scrum es la rapidez con la que se obtienen resultados, gracias a que es una metodología ágil que ayuda a generar entregables en el menor tiempo posible por la alta comunicación que existe entre el cliente y el equipo de desarrollo.

La técnica más eficaz que se empleó en el desarrollo del presente proyecto fue la implementación de las fases de la metodología Scrum que permitieron alcanzar el éxito del proyecto:

\section{Inicio:}

Durante la fase de inicio del proyecto se llevaron a cabo actividades específicas y necesarias para poder tener claro el objetivo del proyecto, que es lo que implica, la fecha de entrega y la formación del equipo. Dichas actividades fueron crear la visión del proyecto, identificar al Scrum master, Stakeholders, crear el Backlog priorizado, crear el equipo Scrum y realizar la planificación de lanzamiento.

\section{Planificación y estimación;}

Para la fase de planificación se llevaron a cabo unas de las actividades más importantes para el proyecto, por ejemplo; crear las historias de usuario, estimar y comprometer las tareas a realizar y crear el Sprint Backlog.

\section{Implementación}

En la fase de implementación se llevaron a cabo todas las actividades a desarrollar para la creación del proyecto, además en esta fase se hicieron las reuniones diarias con un máximo de 15 minutos para resolver dudas y demostración de actividades ya realizadas.

\section{Revisión y retrospectiva}

En la fase de revisión los entregables que fueron desarrollados en la fase de implementación se entregaron al cliente para validar el avance, sugerir cambios y agregarlos al Backlog priorizado para llevarlos en acción.

\section{Lanzamiento}

En esta que es la última fase de la metodología Scrum las actividades que se realizaron fueron enviar los entregables finales al cliente y además se llevó a cabo una revisión y validación del proyecto. Los instrumentos utilizados en el desarrollo del proyecto fueron los mismos que propone la metodología ágil;

- $\quad$ Scrum board: Tablero para establecer las actividades, roles y tiempos en que se realizó cada Sprint.

- Backlog priorizado del producto: lista priorizada de las actividades identificadas en el product Backlog, estas indican el orden en como las actividades se realizarán.

- Product Backlog: lista de actividades a realizar sin priorizar.

\section{Marco teórico}

Norma de seguridad:

NOM-018-STPS-2015, Sistema armonizado para la identificación y comunicación de peligros y riesgos por sustancias químicas peligrosas en los centros de trabajo.

Entró en vigor el 9 de octubre del año 2018. Esta norma establece lineamientos para el manejo de sustancias químicas peligrosas en las empresas, criterios para realizar un sistema armonizado de identificación de riesgos de sustancias químicas peligrosas. En específico, dicho sistema debe incluir lo siguiente: 
- Listado actualizado de las sustancias químicas peligrosas y mezclas. Esto incluye el nombre de la sustancia, su número CAS, y el número CAS de los componentes de cada mezcla. Además, debe contener la clasificación de sus peligros físicos y para la salud.

- $\quad$ Las Hojas de Datos de Seguridad (HDS) de las sustancias químicas peligrosas.

- Señalización o etiquetado.

- Capacitación y adiestramiento a los trabajadores sobre la señalización y las hojas de seguridad.

Según el artículo 9 de la norma en la HDS se deben incluir las sustancias químicas que tengan una concentración igual o mayor a los valores límite de composición en la mezcla. Esto se establece de acuerdo con la clase de peligro que representa para la salud. Entre estos peligros se encuentran la toxicidad aguda, irritación cutánea y en los ojos, sensibilización respiratoria, mutagenicidad, carcinogénica, toxicidad para la reproducción, la específica de órganos blanco en exposición única y repetida. Además, la información descrita debe coincidir con la utilizada en la señalización.

Las HDS son herramientas sustanciales para identificar riesgos $\mathrm{y}$, así, prevenir accidentes en el centro laboral. Esto se complementa con la señalización de las sustancias y la capacitación y adiestramiento a los trabajadores sobre su manejo. Lo anterior, permitirá que las inspecciones realizadas por las autoridades competentes sean aprobadas por las empresas.

Normas relacionadas:

\section{NOM-010-STPS-2014}

Agentes químicos contaminantes del ambiente laboral-Reconocimiento, evaluación y control

\section{NOM-026-STPS-2008}

Colores y señales de seguridad e higiene, e identificación de riesgos por fluidos conducidos en tuberías.

\section{NMX-R-019-SCFI-2011}

Sistema armonizado de clasificación y comunicación de peligros de los productos químicos. (Gaceta, 2016).
Sustancias químicas peligrosas:

Son aquellas que por sus propiedades al ser manejadas, transportadas, almacenadas o procesadas presentan la posibilidad de inflamabilidad, explosividad, toxicidad, reactividad, radiactividad, corrosividad o acción biológica dañina, además de que pueden afectar la salud de las personas expuestas o causar daños a instalaciones y equipos. (NOM-005-STPS1998). (Gaceta, 2016).

Pictogramas de peligrosidad de NOM018-STPS-2015.

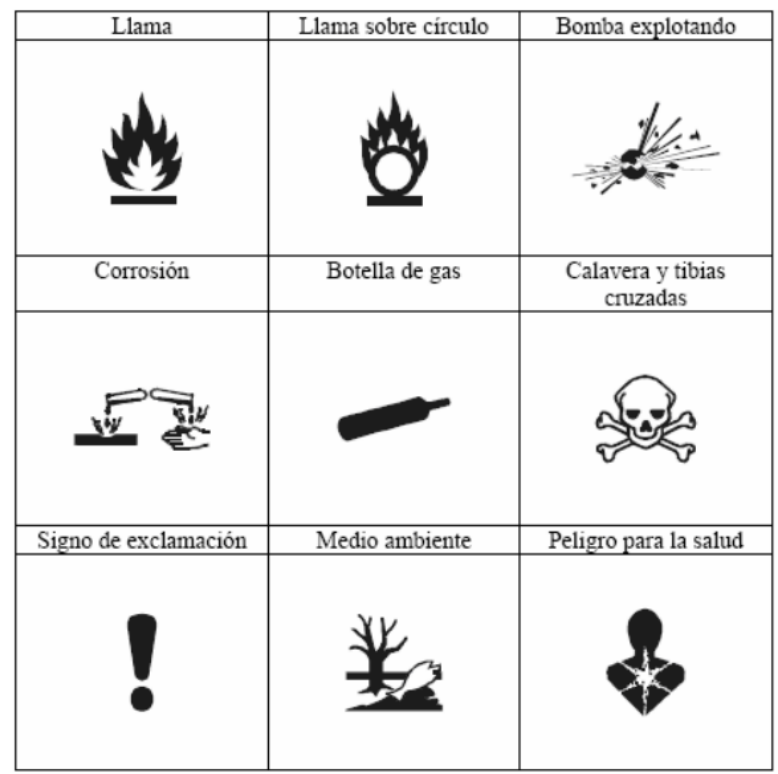

Figura 1 Pictogramas de peligrosidad (Diario Oficial de la Federacion, 2015)

Recomendaciones para manejo de sustancias.

El manejo de las sustancias químicas peligrosas está establecido en la Hoja de Datos de Seguridad (HDS) que cada sustancia debe tener. Es de suma importancia que cuando se adquiera alguna de éstas se solicite al proveedor este documento sin excusa alguna, ya que en él se describen todas las precauciones que se deben tomar cuando se manipulan las sustancias. (Gaceta, 2016)

Antecedentes de aplicaciones: 


\section{Drager VOICE:}

Es una aplicación móvil similar a la de Solucion.Es la cual su objetivo principal es la consulta de las sustancias químicas que existen en el mundo, cuenta con una lista de más de 1.700 sustancias peligrosas y 11.500 sinónimos está ahora disponible en diferentes idiomas e informa sobre los valores límite locales.

\section{Emergency Response Guide 2016 (NIH/NLM):}

El ERG contiene una lista indexada de productos peligrosos y el número de ID asociado, los peligros generales que plantean y las precauciones de seguridad recomendadas. Por ejemplo, si el personal de emergencia llega a la escena de un remolque volcado que muestra una pancarta de materiales peligrosos del DOT, usaría la guía para identificar el material asociado con el cartel y recibir orientación sobre cómo responder en consecuencia.

\section{TOXNET Mobile (NIH/NLM):}

Grupo de bases de datos que abarca los productos químicos y las drogas, las enfermedades y el medio ambiente, la salud ambiental, la seguridad y salud en el trabajo, el envenenamiento, la evaluación y reglamentación de los riesgos y la toxicología.

\section{NIOSH Pocket Guide to Chemical Hazards:}

Guía de bolsillo de NIOSH sobre peligros químicos. Esta aplicación fue desarrollada y aprobada por el Instituto Nacional de Seguridad y Salud Ocupacional (NIOSH), dentro del Departamento de Salud y Servicios Humanos de EE. UU.

Pathogen Safety Data Sheet (Public Health Agency of Canada):

Las hojas de datos de seguridad de patógenos (PSDS, por sus siglas en inglés) son documentos técnicos que describen las propiedades peligrosas de un patógeno humano y recomendaciones para el trabajo que involucra a estos agentes en un entorno de laboratorio. Estos documentos han sido producidos por la Agencia de Salud Pública de Canadá como recursos educativos e informativos para el personal de laboratorio que trabaja con estas sustancias infecciosas.

\section{Manejo de sustancias}

El inadecuado manejo y la acumulación de los residuos sólidos, en las zonas urbanas rompen el equilibrio ecológico y dinámico del ambiente generando contaminación al ambiente y a la salud de las personas. (Miraval, E., \& Kunder, J. 2019).

\section{Tecnología:}

\section{Aplicaciones móviles}

Una aplicación móvil es una aplicación informática diseñada para ser ejecutada en teléfonos inteligentes, tabletas y otros dispositivos móviles. Las aplicaciones permiten al usuario efectuar un conjunto de tareas de cualquier tipo profesional, de ocio, educativas, de acceso a servicios, etc., facilitando las gestiones o actividades a desarrollar

\section{Android}

Android es un sistema operativo basado en Linux que fue pensado en un principio para usarse con un teclado y un cursor que permitía navegar por las aplicaciones. En la actualidad, tras varias actualizaciones, está pensado para dispositivos móviles con pantalla táctil, ya sean smartphones o tabletas. Inicialmente fue creado por la compañía de software Android Inc., pero en el año 2005 Google compró la empresa y 2 años después presentó el sistema operativo.

El hecho de que convierta cualquier teléfono en prácticamente un ordenador de bolsillo lo hace cómodo para los usuarios, y que sea de código abierto facilita las cosas a fabricantes y desarrolladores. Hacer aplicaciones para su uso en un dispositivo móvil, o su instalación en uno de ellos, no tiene ningún coste, por lo que lanzar un teléfono o aplicación con Android tiene un bajo coste. Además, que sea libre y cualquiera pueda inspeccionar su código fuente facilita que se detecten los fallos más rápidamente y que los fabricantes puedan adaptar de una manera más sencilla el sistema operativo a sus terminales con el objetivo de ofrecer más posibilidades a sus usuarios. Otros sistemas operativos funcionan con un código cerrado en el que sólo el fabricante del mismo puede hacer modificaciones. 


\section{Android Studio}

Android Studio es el entorno de desarrollo integrado (IDE) oficial para el desarrollo de aplicaciones para Android y se basa en IntelliJ IDEA. Además del potente editor de códigos y las herramientas para desarrolladores de IntelliJ, Android Studio ofrece aún más funciones que aumentan tu productividad durante la compilación de apps para Android, como las siguientes:

- Un sistema de compilación basado en Gradle flexible

- $\quad$ Un emulador rápido con varias funciones

- Un entorno unificado en el que puedes realizar desarrollos para todos los dispositivos Android

- Instan Run para aplicar cambios mientras tu app se ejecuta sin la necesidad de compilar un nuevo APK

- Integración de plantillas de código y GitHub para ayudarte a compilar funciones comunes de las apps e importar ejemplos de código

- Gran cantidad de herramientas y frameworks de prueba

- Herramientas Lint para detectar problemas de rendimiento, usabilidad, compatibilidad de versión, etc.

- $\quad$ Compatibilidad con C++ y NDK

- $\quad$ Soporte incorporado para Google Cloud Platform, lo que facilita la integración de Google Cloud Messaging y App Engine

\section{Bases de datos}

Es un conjunto de datos pertenecientes a un mismo contexto y almacenados sistemáticamente para su posterior uso. En este sentido, una Biblioteca puede considerarse una base de datos compuesta en su mayoría por documentos y textos impresos en papel e indexados para su consulta.

En la actualidad, y debido al desarrollo tecnológico de campos como la Informática y la Electrónica, la mayoría de las bases de datos están en formato digital (electrónico), que ofrece un amplio rango de soluciones al problema de almacenar datos.
Existen programas denominados sistemas gestores de bases de datos; los sistemas de gestión de bases de datos son un tipo de software muy específico, dedicado a servir de interfaz entre la base de datos, el usuario y las aplicaciones que la utilizan.

\section{SQLite}

En Android existen varias formas, sobre cómo guardar datos de forma persistente.

Entre los métodos más comunes encontramos:

- $\quad$ Bases de datos SQLite

- $\quad$ SharedPreferences

- $\quad$ Almacenamiento en disco

Guardar datos de forma persistente significa que los datos persisten incluso si se apaga y se vuelve a prender el dispositivo.

Generalmente cuando cerramos una aplicación, Android va a liberar los recursos que ésta tenía asignados.

Nuestras variables que estaban definidas dejarán de existir. Es por eso que necesitamos guardar estos datos si son importantes.

$\mathrm{Y}$, hay que tener en cuenta que estos datos se están registrando de forma local.

Es decir, estas 3 opciones almacenan los datos en el mismo dispositivo, sin requerir de una conexión a internet.

\section{Java}

Java es un lenguaje de programación de propósito general, concurrente, orientado a objetos, que fue diseñado específicamente para tener tan pocas dependencias de implementación como fuera posible. Su intención es permitir que los desarrolladores de aplicaciones escriban el programa una vez y lo ejecuten en cualquier dispositivo, lo que quiere decir que el código que es ejecutado en una plataforma no tiene que ser recompilado para correr en otra. Java es, a partir de 2012, uno de los lenguajes de programación más populares en uso, particularmente para aplicaciones de cliente-servidor de web, con unos diez millones de usuarios reportados. 


\section{Desarrollo}

\section{Diagramas UML}

\section{Casos de Uso-Administrador}

En la siguiente imagen se puede apreciar cuales son los casos de uso llevados a cabo por parte del usuario que será el administrador de la aplicación.

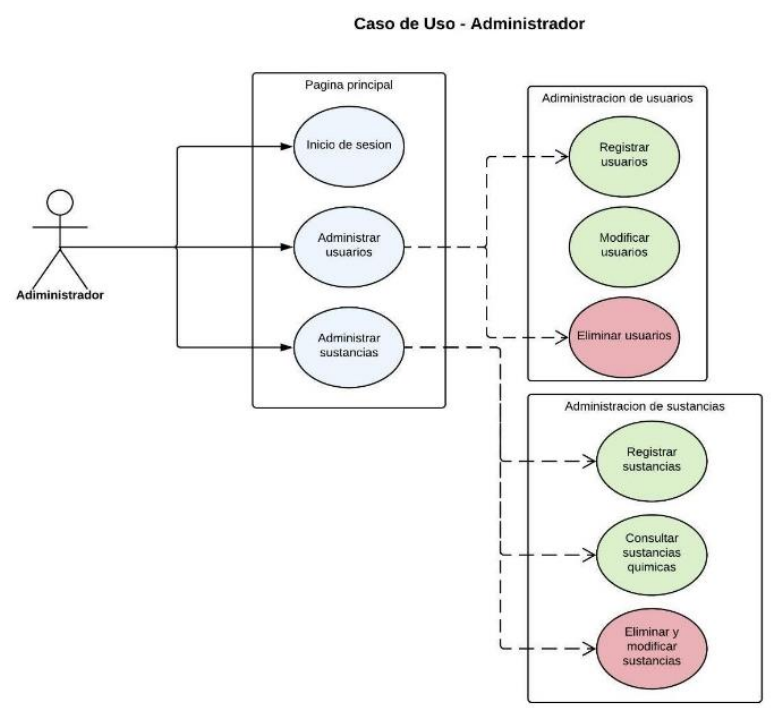

Figura 1 Caso de uso administrador

\section{Caso de uso - Usuario común}

En la siguiente imagen se puede apreciar cuales son los casos de uso llevados a cabo por parte del usuario común.

Caso de uso - Usuario común

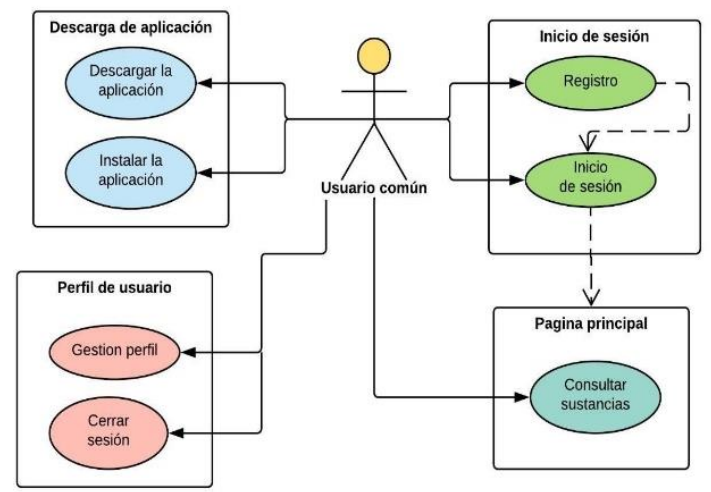

Figura 2 Usuario común

\section{Interfaz grafica para el usuario}

\section{Pantalla principal (HOME)}

La siguiente imagen que se presenta es la pantalla princiapl de la aplicación web la cuenta con el nombre la aplicación, el logo y ademas cuenta con un boton en la esquina superior izquierda para poder acceder al menu lateral.

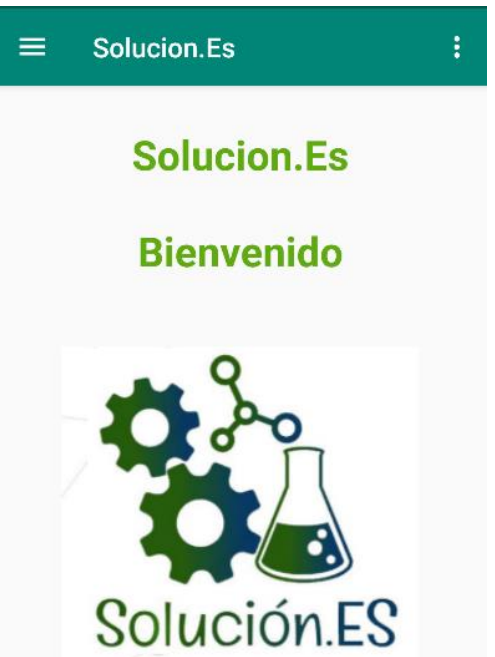

Figura 3 Pantalla principal

\section{Menú lateral}

A continuación, se puede visualizar el menú lateral el cual muestra todas las opciones a las que podemos acceder.

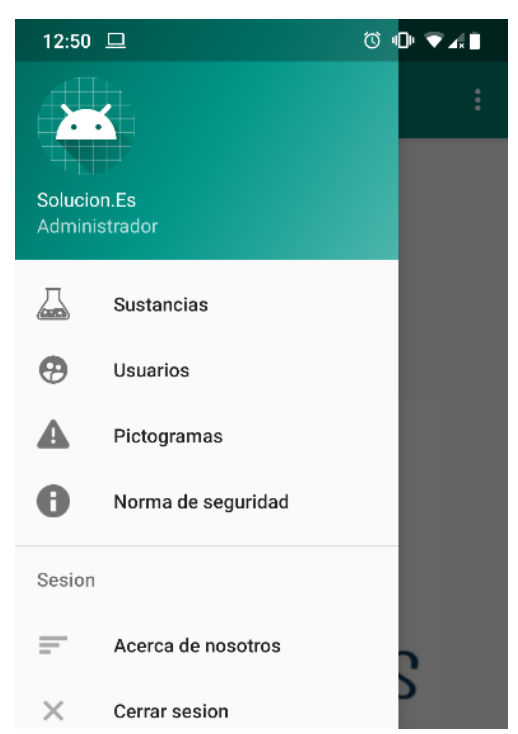

Figura 4 Menú lateral 


\section{Seccion de sustancias}

En la imagen de acontinuacion se muestra cuales son la opciones u operaciones que se pueden realizar para seccion las sustancias quimicas.

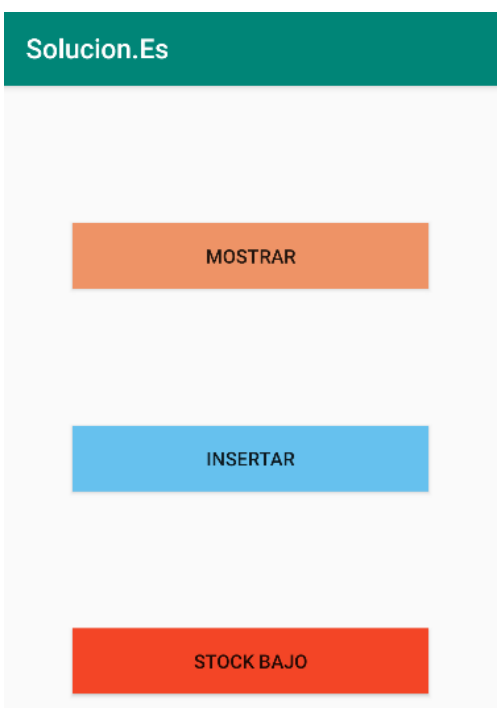

Figura 5 Sección de sustancias

\section{Insertar sustancias}

En la pantalla siguiente se muestra la pantalla de insertar sustancias donde debemos llenar todos los campos de nueva sustancia.

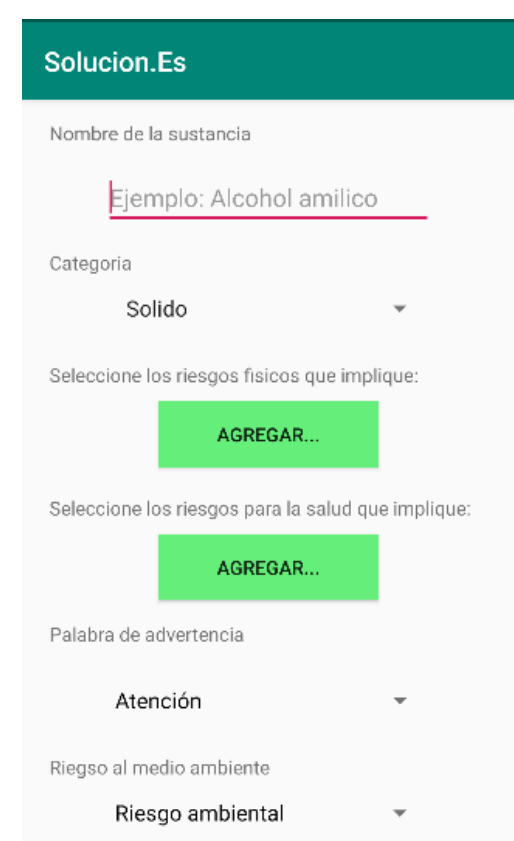

Figura 6 Insertar sustancias

\section{Lista de sustancias}

En la pantalla de a continuacion se muestra el apartado donde podemos visualizar todas las sustancias que han sido agregadas o estan en existencia.

\section{Solucion.Es}

\section{Lista de Sustancias}

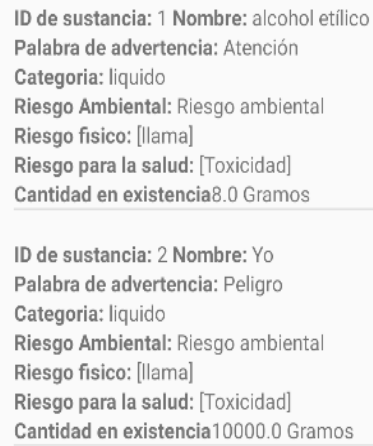

Figura 7 Lista de sustancias

\section{Stock bajo de sustancias}

En la pantalla siguiente se muestra la sección de sustancias que tiene un stock bajo en el almacén previamente establecido,

\section{Solucion.Es}

\section{Sustancias por Adquirir}

Nombre: alcohol etilico

Cantidad en existencia: 8.0 Gramos/Mililitros

Nombre: $g$

Cantidad en existencia: 1.0 Gramos/Mililitros

Nombre: hkahx

Cantidad en existencia: 6.0 Gramos/Mililitros

\section{Figura 8 Stock bajo}

\section{Pictogramas de peligrosidad}

En la pantalla de a continuacion se muestra la pantalla de los pictogramas de peligrosidad que existen para el etiquetado de las sustancias quimicas. 


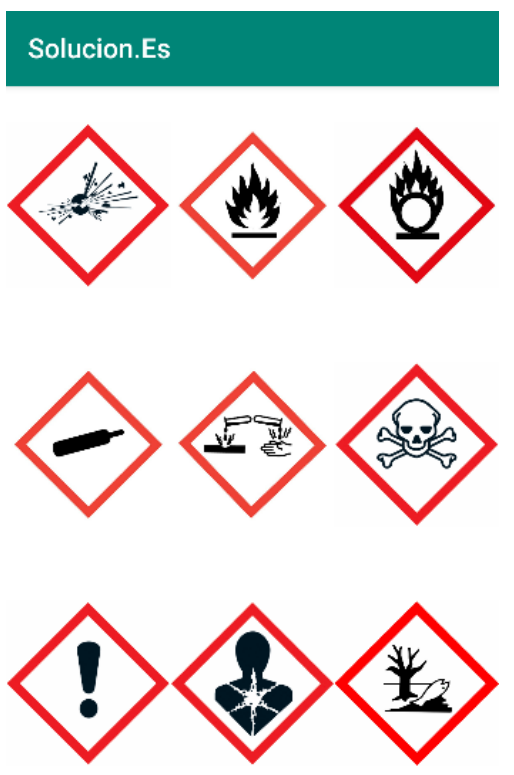

Figura 9 Pictogramas de peligrosidad

\section{Norma de seguridad}

En la siguiente imagen se muestra la pantalla que existe dentro de la aplicación que muestra información acerca de la norma de seguridad implementada en el almacén.

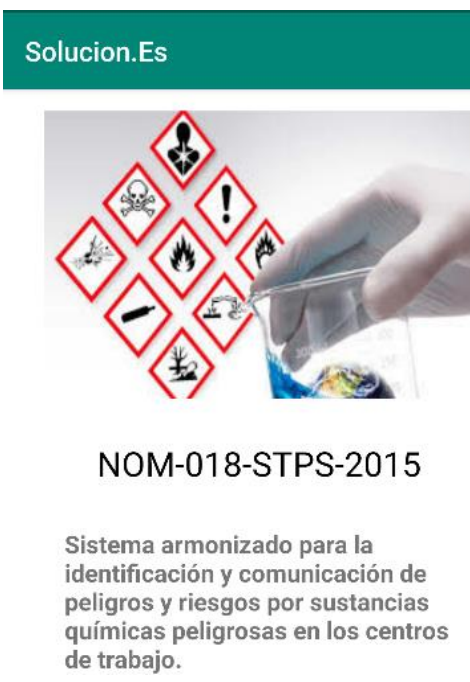

Figura 10 Norma de seguridad

\section{Acerca de nosotros}

En pantalla siguiente se logra visualizar información acerca de cuál fue el motivo de la aplicación móvil y además muestra información de los desarrolladores.

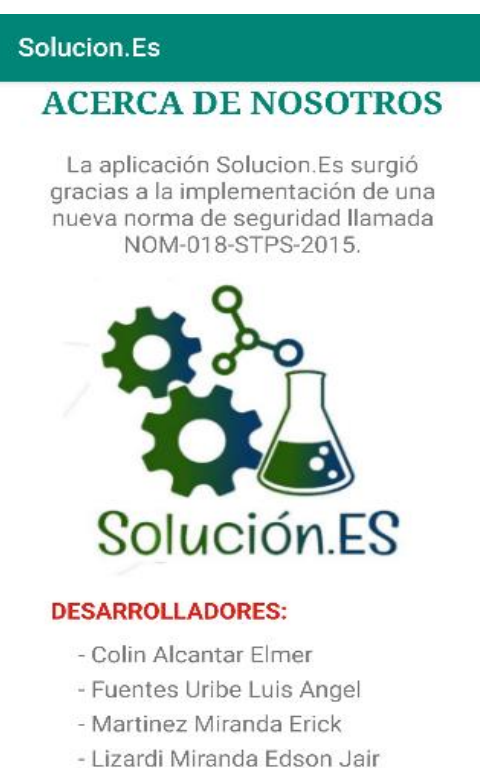

Figura 11 Acerca de nosotros

\section{Resultados}

Para obtener resultados precisos acerca del funcionamiento de la aplicación móvil se aplicó una encuesta a una muestra poblacional de 51 personas $92 \%$ fueron alumnos y $6 \%$ docentes y $2 \%$ laboratoristas del área de Tecnologías de la información y Química.

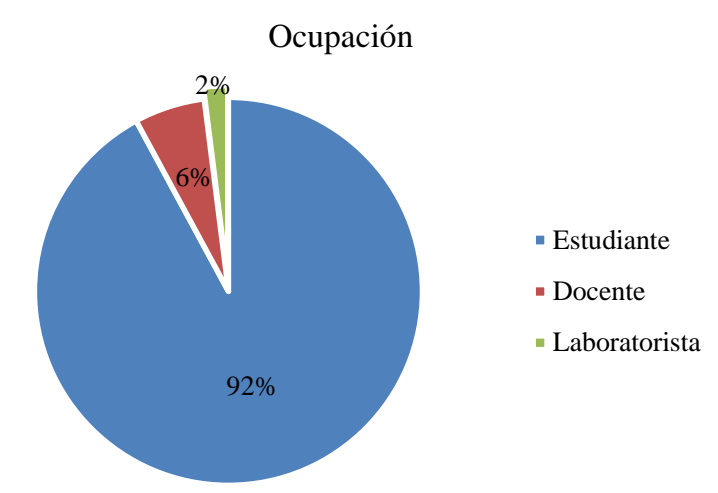

Grafica 1 Muestra poblacional

La encuesta está dividida en tres secciones que permitieron identificar diversos factores de calidad de la aplicación y en qué grado se cumple.

1. Interfaz del sistema: Preguntas acerca del diseño, usabilidad y facilidad de navegación. 
¿ Le gustan los colores de la interfaz de la aplicación?

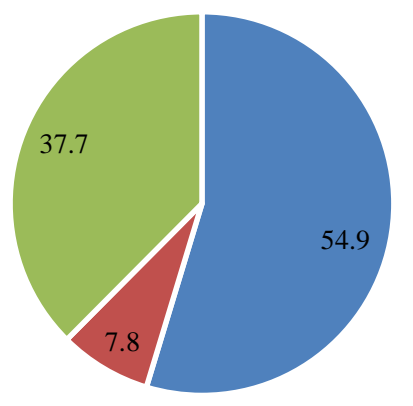

- Son agradables

- Me es indiferente

- Demasiado

Grafica 2 Factores de calidad-color

¿Considera que es fácil utilizar la aplicación?

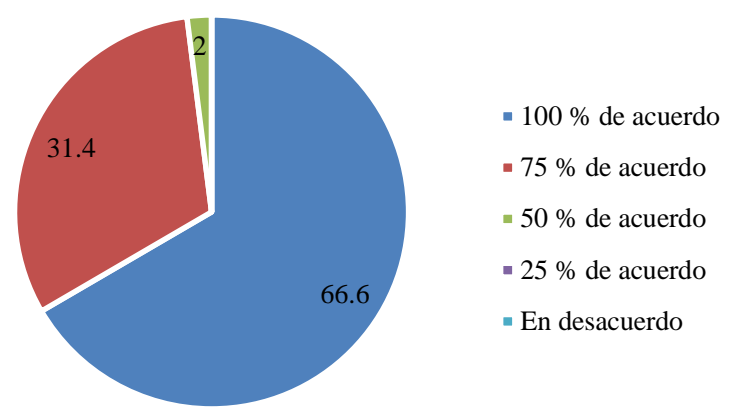

Grafica 3 Factor de calidad - usabilidad.

¿La barra lateral facilita la navegación?

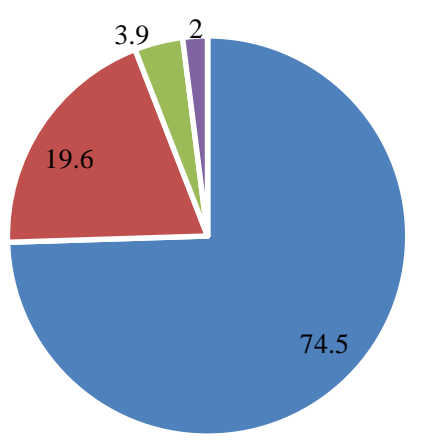

- $100 \%$ de acuerdo

- $75 \%$ de acuerdo

- $50 \%$ de acuerdo

- $25 \%$ de acuerdo

- En desacuerdo

Grafica 4.- Factor de calidad - Navegación.

¿La aplicación cuenta con un logotipo?

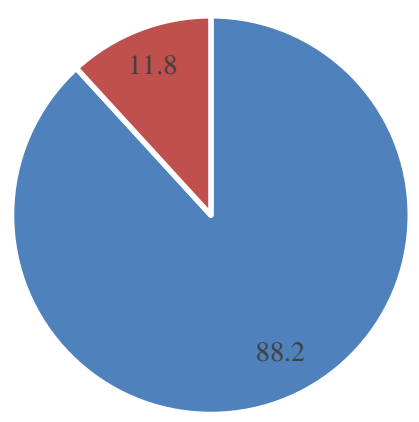

- $\mathrm{Si}$

- No

Grafica 5 Factor de calidad - Diseño
¿La aplicación muestra información acerca de la norma NOM-018-STPS-2015 ?

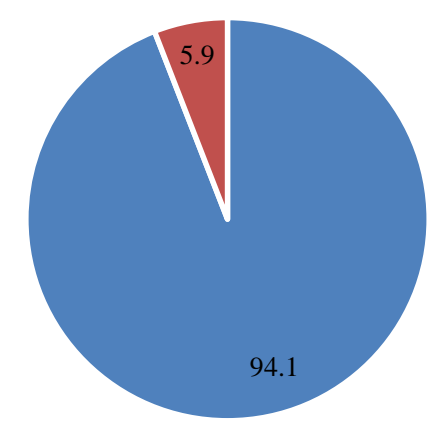

- Si

- No

Grafica 6 Información importante.

1. Funcionalidad de la aplicación: Preguntas acerca del cumplimiento de los requerimientos funcionales (inserciones, modificaciones, eliminaciones, consultas, etc.) establecidos por el cliente (Docentes y alumnos del área de Química).

¿La aplicación permite realizar inserciones a una base de datos?

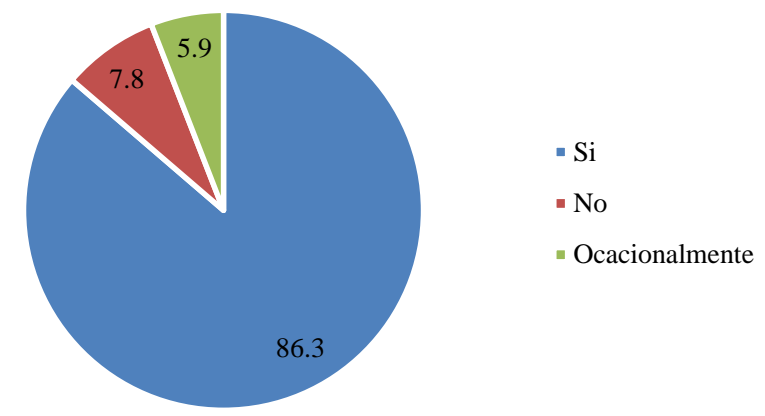

Grafica 7 Requerimiento funcional - Inserción.

¿La aplicación permite realizar modificaciones sobre los registros de la base de datos?

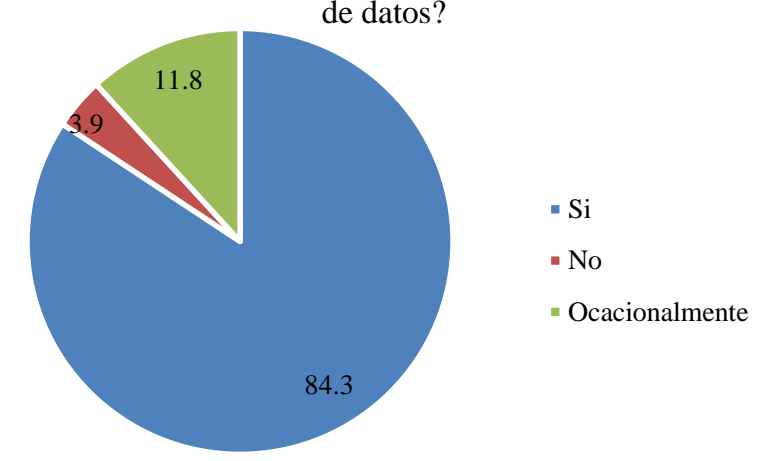

Grafica 8 Requerimiento funcional - modificación

ARCIGA-PEDRAZA, Raquel, FUENTES-URIBE, Luis Ángel, JUAREZ-SANTIAGO, Brenda y MARTINEZ-MIRANDA, Erick. Desarrollo de una Aplicación Móvil para la eficiente gestión de sustancias Químicas con base en la Norma Oficial Mexicana NOM-018STPS-2015. Revista de Cómputo Aplicado. 2019. 
¿La aplicación permite visualizar los datos registrados?

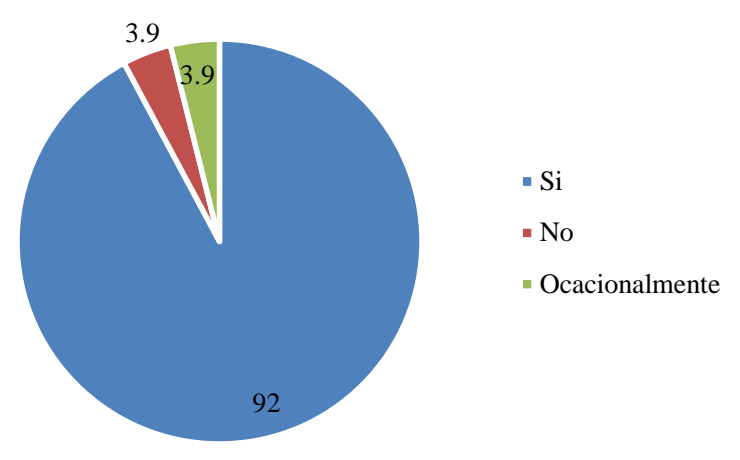

Grafica 9 Requerimiento funcional - consultas.

¿La aplicación permite eliminar los datos registrados?

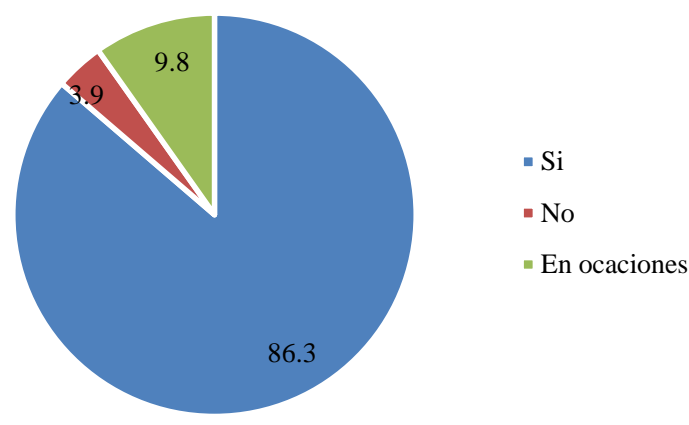

Grafica 10. Requerimiento funcional - eliminar.

2. Solución a la problemática.

¿La aplicación realmente resolvió un problema?

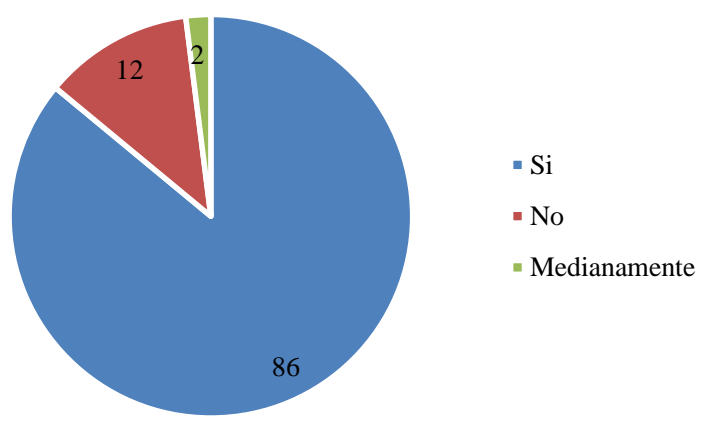

Grafica 11 Resolución del problema.

\section{Agradecimiento}

El equipo de trabajo, agradecemos a la Universidad Tecnológica de San Juan del Río por financiar los gastos económicos que implico la participación del presente proyecto en el marco de la 4ta. Edición del Congreso Interdisciplinario de Energías Renovables, Mantenimiento Industrial, Mecatrónica e Informática. (CIERMMI 2019).

\section{Conclusiones.}

El desarrollo e implementación de la aplicación para los laboratorios de la UTSJR en efecto trajo consigo grandes mejoras en el proceso de gestión y control de inventario de las sustancias utilizadas puesto que la información ahora está centralizada, integra, disponible y con un alto grado de portabilidad los 365 días del año en comparación con el proceso anteriormente utilizado, del mismo modo con base en los resultados obtenidos es posible afirmar que el $98 \%$ de los usuarios consideran que es fácil utilizar la aplicación en un $87.5 \%$, siendo un indicador positivo, así mismo es funcional en un $87.2 \%$ en cuanto a la ejecución de las operaciones básicas (insertar, modificar, eliminar, consultar) para las cuales ha sido desarrollada

\section{Referencias}

Diario Oficial de la Federacion. (09 de 10 de 2015). Diario Oficial de la Federacion. Obtenido de Diario Oficial de la Federacion: http://www.dof.gob.mx/nota_detalle.php?codig $\mathrm{o}=5411121 \&$ fecha $=09 / 10 / 2015$

Ecured. (s.f.). Ecured. Obtenido de Ecured: https://www.ecured.cu/Bases_de_datos

Gaceta. (03 de 06 de 2016). http://www.olade.org. Obtenido de http://www.olade.org:

http://www.olade.org/realc/docs/doc_103799_2 0170515115149.pdf

Pevencionar.com.mx. (09 de 10 de 2018).

Pevencionar.com.mx. Obtenido de

Pevencionar.com.mx:

http://prevencionar.com.mx/2018/10/09/ultimahora-entro-en-vigor-la-nom-018-stps-2015/ 
Programacion y mas. (s.f.). Programacion y mas. Obtenido de Programacion y mas: https://programacionymas.com/blog/bases-dedatos-sqlite-en-android

Rastreador.com. (Abril de 2014). Obtenido de Rastreador.com:

https://www.rastreator.com/telefonia/articulosdestacados/el-sistema-operativo-android.aspx

Redciatox. (219). Redciatox. Obtenido de Redciatox:

https://www.redciatox.org/aplicaciones/aplicaci ones-moviles

Sinec. (s.f.). Sinec. Obtenido de Sinec: https://www.sinec.gob.mx/SINEC/Vista/Norma lizacion/DetalleNorma.xhtml?pidn=MjZRODB xNk0wVTZNWkd4M2ZmczhxUT09

Miraval, E., \& Kunder, J. (2019). SISTEMA DE CONTROL CON CÓDIGOS QR PARA LA MEJORA DE LA GESTIÓN DE RESIDUOS SÓLIDOS INORGÁNICOS APROVECHABLES DE LOS DOMICILIOS DE CAYHUAYNA ALTA PILLCO MARCA, HUÁNUCO NOVIEMBRE-2018-ENERO2019 